\title{
Young people's perceptions of mental and physical health in the context of general wellbeing
}

Joanne H. Singletary ${ }^{a}$, Craig L. Bartle ${ }^{a}$, Nadzeya Svirydzenka ${ }^{b}$, Nicola M. Suter-Giorgini ${ }^{a}$, Annette M. Cashmore ${ }^{a}$ and Nisha Dogra ${ }^{b}$

${ }^{a}$ GENIE Centre for Excellence in Teaching and Learning, University of Leicester, UK

${ }^{\mathrm{b}}$ The Greenwood Institute of Child Health, University of Leicester, UK

\begin{abstract}
Objectives: Recognition of the need for health education in schools has seen advances in health literacy in recent years. Most of these have focused on physical health whereas education about mental health is generally lacking and focused on tackling stigma rather than promoting good mental health. This study evaluated a pilot intervention designed to improve young people's understanding of good mental health as a key aspect of wellbeing and explores their perceptions of health and wellbeing.

Methods: Two hundred and eighteen 13-year-olds participated in an interactive workshop about healthy eating, physical activity and mental health. Young people's understanding and perceptions were assessed through anonymous questionnaires at the start and end of the workshop. Common themes were identified and differences pre- and post-workshop and between girls and boys were analysed.

Results: Nearly all young people (100\% before, $97 \%$ after) perceived being healthy to mean being physically healthy. A minority (8\%) also considered mental health to be a component of general health, which increased to $12 \%$ after the workshop. Understandings of mental health broadened after the workshop. Interestingly many mentioned physical health when asked to describe mental health, both before and after the workshop. Girls' and boys' responses were similar except for more girls including social relationships in their descriptions of mental health.

Conclusions: In this study, we have discovered more about how young people perceive health in general and mental health. Such information is useful for targeting future interventions. Students' understanding of mental health and its importance to wellbeing can be improved through short combined health interventions.
\end{abstract}

\section{Keywords}

Adolescent health, health education, mental health, attitudes to health, intervention

\section{Corresponding author:}

Nicola Suter-Giorgini, GENIE CETL, Department of Genetics, University of Leicester, University Road, LE1 7RH. Email: nms2@le.ac.uk 


\section{Introduction}

Education about mental health, as opposed to mental illness, is becoming a growing priority as understanding of its contribution to health and wellbeing increases. One in four people will experience mental health problems at some point during their life (WHO, 2003) and mental health disorders account for three of the ten leading causes of disease burden in high-income countries (Lopez et al., 2006). It is widely acknowledged that $25 \%$ of children and young people, experience mental health problems and of these $10 \%$ experience mental illness although this can vary across different groups (WHO, 2005; Green et al., 2005; Dogra et al., 2013). Like physical health, mental health can be improved through increased awareness of what it is to be mentally healthy and through taking steps to safeguard mental health (Jané-Llopis et al., 2005). It is well established that mental health and physical health are interlinked and a recent NHS England report, No Health without Mental Health highlights the need for parity between mental and physical health and to see both as integral components of overall health ( $\mathrm{DoH}, 2011)$. Associations between childhood behavioural and mental health problems and premature mortality have been observed (Jokela et al., 2009), as have associations with diabetes, obesity and poorer general health and wellbeing (von Stumm et al., 2011; White et al., 2012). These associations and increased risk factors may be due in part to the development of poor adult health-related habits and the promotion of childhood and adolescent mental health may be an important component of disease prevention strategies (White et al., 2012).

The need for health interventions targeted towards adolescents and young people is becoming increasingly clear as efforts continue to address high levels of obesity, low levels of physical activity, and high levels of stigma towards those with mental health problems (e.g. (Pinfold et al., 2003; Brown and Summerbell, 2009)). Reports from the English Government (DCSF, 2009; DES, 2005) and the World Health Organization (WHO, 1997) suggest the need to educate young people and facilitate their ability to make responsible choices for themselves and their health, a need that has long been recognised when addressing physical health (Brown and Summerbell, 2009). However, there has been little research into the effectiveness of interventions educating about mental health, despite relatively high levels of negative attitudes in young people and adults in the UK ((Friedli, 1999; Watson et al., 2004; Armstrong et al., 2000) and others). Additionally, little is known about how young people perceive mental health and health in general, in regards to themselves and others, but it has been suggested that their perceptions of mental health differ from those expected by adults and policy makers (Naylor et al., 2009). A limited amount of research has shown views of health to be broader than just physical health for United States (US) (Ott et al., 2011; Natapoff, 1978), Australian (Ward and Wang, 1997) and Chinese (Wang, 2004) young people, but assessment of unprompted beliefs about health remains relatively understudied.

Most research into school-based mental health education interventions has focused on changing negative attitudes and reducing stigma, with the additional desired outcome of improving self-efficacy in seeking help for mental health problems. In this study we attempted a different approach and followed two main objectives. First, we aimed to establish knowledge-base and perceptions of secondary school students about general physical and mental health and wellbeing in order to identify the gaps and barriers to inform targeted interventions addressing these gaps in the future. Second, we wanted to evaluate the success of a short interactive intervention designed to stimulate discussions and knowledge exchange about mental and physical health. The success of the intervention 
would be evidenced by increased understanding of health issues and knowledge change in participating students.

\section{Methods}

\section{Participants}

Two hundred and eighteen Year 9 (age 13-14) young people from five schools in Leicestershire took part in a four hour long event titled 'Healthy body, healthy mind' held at the University of Leicester. The event was run twice, on two consecutive days (101 students attended day 1 and 117 day 2). The mean age of participants was 13.08 years (SD=0.28) and the sample consisted of $54.1 \%$ female $(\mathrm{N}=118)$ and $40.4 \%$ male $(\mathrm{N}=88)$ participants, with $5.5 \%$ not providing gender information $(\mathrm{N}=12)$.

31 schools that had previously attended events run by the university received invitations to the event. The first five schools that responded were included in the study. Each school decided how many students to bring, with final numbers varying from four to 113 students per school. The school that brought four students did so as the rest of the year group had participated in a similar event previously. The students were generally of mixed ability but one school only offered the opportunity to attend to their top stream of classes, that is the higher performing students ( 40 in total). The schools were all rural or sub-urban state-run, coeducational middle schools (ages 11-14) in the county of Leicestershire, UK. Three of the schools (30\% of the total participants) had predominantly White students and two of the schools (70\% of the total participants) had approximately one quarter Asian or Mixed Asian/White students and two thirds White. The overall ethnicity breakdown was 75.7\% White, 17\% Asian, 5.4\% Mixed, 0.6\% Black, 0.3\% Chinese, and 1.2\% Other.

\section{Intervention}

The intervention took the form of a four hour long event held at the University of Leicester in multidisciplinary laboratories often used for outreach and public events. The event consisted of an introduction and preliminary questionnaire, two workshops (separated by lunch) and a second questionnaire. Workshop 1 was an 85 minute session of interactive activities about healthy eating and physical health ('Healthy Body') and workshop 2 was a 45 minute session exploring the meaning of the term 'Mental Health' and what helps young people to stay mentally healthy ('Healthy Mind'). Half of the students participated in workshop 1 first and half in workshop 2 first. All the students participated in both workshops. The findings and exploration of students' perceptions from the mental health workshop are described separately (Svirydzenka et al., 2014).

The 'Healthy Body' workshop was developed by JS and NS-G and was based on activities and materials previously used with other 12-13 year old students as part of a wider Health Education Reaching Out (HERO) programme - a programme looking at the best ways to educate school students about health eating and physical activity. The activities took the form of stations that students could visit at their own pace and order of their choice during the session. Some of the activities were self-directed and others were facilitated by staff and university students from GENIE that had previous experience of delivering these activities to this age group. The content of the workshops is described in Box 1. 
The 'Healthy Mind' workshop was developed by ND and NS and the content of the workshops is also described in Box 1 (Svirydzenka et al., 2014).

\section{Ethics}

The schools obtained written parental consent for students to attend the event, in line with the schools' own procedures for taking students off-site. The students attended as part of their schooling. The use of questionnaires was approved by the University of Leicester Committee for Research Ethics Concerning Human Subjects (Non-NHS) as part of the Health Education Reaching Out project. Students were free to not take part in an activity or to not complete the questionnaire if they wished.

\section{Measurements}

To explore students' perceptions and understanding of both general health and mental health students were asked to complete the same anonymous paper-based questionnaire on arrival to the event before commencing the activities and again at the end of the event before leaving. ID numbers were not issued for ease of administration and as such pre- and post-event questionnaire responses were not linked. There was no further follow up. The students all completed the questionnaire at the same time and in the same room. The questionnaire consisted of a series of free-text response questions followed by some Likert scale questions that are not reported here. To investigate whether young people perceive mental health as being part of general health students were asked to describe firstly 'what makes someone healthy' by listing things they thought were important for general health, and secondly 'what makes you mentally healthy' by listing things they thought were important for mental health. Responses to both questions were as free text. Questions about individual demographics were limited to age and gender to keep the questionnaire short. Young people were invited to give feedback on the questionnaire for its accessibility. Minor modifications were made in response to a questionnaire piloted with 35 12-13 year olds in a sub-urban state-run, mixed gender middle school that did not receive the intervention.

\section{Analysis}

The open response questions were analysed using content analysis to identify categories within the data by three of the team independently and then censuses reached on the themes identified. The majority of responses were then categorised into either 'physical health-related' or 'mental health-related'. The remaining responses were categorised as being 'related to both physical and mental health'. This category contained two types of response: one or more topic relating to each category was listed, or the topic listed relates to both physical and mental health (such as avoiding intoxicants and getting enough sleep). An identical coding frame was used to code both sets of responses (pre and post) making it possible to identify any statistically significant differences between before and after the event and between male and female students using Pearson Chi Square Test for Independence with Yates' Continuity Correction. Fisher's Exact Probability Test was used where there were more than $20 \%$ of expected frequencies less than 5 . A probability less 
than 0.05 was regarded as statistically significant. Statistical analyses were conducted using IBM SPSS Statistics 20.

\section{Results}

Response rates

All 218 students completed the questionnaire before the event. 214 students (98\%) completed the questionnaire after the event. Of the completed questionnaires, the average response rate for the questions analysed in this paper was $98 \%$ before the event (average for boys $95 \%$, average for girls $99 \%$, average for students who didn't give their gender $100 \%$ ) and $93 \%$ after the event (boys $94 \%$, girls $98 \%$, unknown gender $80 \%$ ).

\section{Perceptions and understanding of general health and mental health}

By beginning the questionnaire with a question about general health we aimed to see if students focussed more on 'physical' or 'mental' health before we introduced either topic.

Before the event, all students $(\mathrm{N}=218)$ mentioned physical health-related topics, such as 'exercising regularly' and 'eating healthily', with $8 \%$ (17) also mentioning mental health-related topics, such as 'having a high self esteem or positive attitude', 'engaging in mentally stimulating activities' and 'having good social relationships', and 25\% (54) mentioning topics that were related to both physical and mental health, such as 'getting enough fresh air or sunlight' and 'avoiding intoxicants'. No students mentioned only mental health-related topics. After the event there was a 3\% decreases in students mentioning physical health-related topics ( $P=0.017)$, corresponding with a $4 \%$ increase in students mentioning mental health-related topics in addition to physical health-related topics (not significant). Results for boys and girls were similar for both the physical health-related and mental health-related categories but differed for the combined category with significantly more girls mentioning topics relating to both physical and mental health before the event $(\mathrm{P}=0.001)$.

Most answers relating to physical health fell into two categories: 'eating healthily and having a balanced diet' (98\%/214 student before teaching, 85\%/173 after) and 'taking regular exercise' (92\%/201 students before teaching and 82\%/167 students after), whereas there was more variety for the few answers relating to mental health (e.g. 'having a high self-esteem', 'positive thinking/attitudes', 'avoiding stressful situations', 'being organised/thinking clearly', 'engaging in mentally stimulating activities and pursuits', and 'having good social relationships and support networks'). Examples of post-event responses relating to mental health are given in Table 3.

The responses to the question 'what makes you mentally healthy' were grouped into five main categories: responses relating to physical health, social relationships, mentally stimulating activities, positive and rational thinking, and avoiding stressful situations. Before the event a third of students (35\%/72 students) referred to mentally stimulating activities (such as learning, keeping busy, participating in activities that give you pleasure, and limiting screen time). This decreased to $23 \% / 44$ students after the event $(P=0.011)$ as the students broadened their views with the increased reference to what was taught in the mental health workshop. Interestingly, $43 \%$ of students (90) mentioned physical health-related topics (such as exercise, rest, and eating a balanced diet) before the event with similar figures after 
the event, indicating a prior understanding of mental health being linked to physical health, which was maintained. One third of students referred to positive and rational thinking (such as having high self-esteem, being open minded, and understanding and handling emotions), a key topic covered in the workshop, before the event (33\%/69 students), increasing to $57 \%$ (111 students) after the event $(\mathrm{P}=0.000)$. There was also an increase in students referring to social relationships (such as having good social skills, a good support network and feeling able to talk openly; $20 \% / 41$ students increasing to $37 \% / 72$ students, $\mathrm{P}=0.000$ ) and those mentioning more than one topic (44\%/92 students increasing to $62 \% / 120$ students, $\mathrm{P}=0.001$ ), indicating a broadening and deepening of understanding following the mental health workshop. Example responses relating to 'positive and rational thinking' and 'social relationships' are given in Table 4. Response patterns for boys and girls (Table 2) were largely similar except for the higher percentage of girls mentioning topics related to social relationships before (girls $27 \% / 31$, boys $9 \% / 7, \mathrm{P}=0.003$ ) and after the event (girls $48 \% / 49$, boys $22 \% / 13, \mathrm{P}=0.002$ ).

\section{Discussion}

This study aimed to investigate young people's perceptions of health, particularly mental health, and to investigate the effect of a one-off educational intervention delivered in school time on students' understanding of health and wellbeing (as opposed to illness). This is a relatively under-studied area of research, especially compared to the number of studies aiming to address mental illness stigma. We sought to investigate perceptions of health and mental health without imposing a specific model answer.

\section{Baseline views about health}

Unsurprisingly, and in keeping with the findings of others (Ott et al., 2011; Wang, 2004; Natapoff, 1978), when asked to describe what makes someone healthy all students focused largely on physical health-related topics. The vast majority of responses related to eating healthily and exercising, consistent with previous work (Ward and Wang, 1997) and the UK (Grace et al., 2012). This is likely to be a reflection of government public awareness campaigns in the UK in recent years and the UK Healthy Schools Programme, which have focussed on healthy eating and activity in schools and families (DoH, 2010; Bremner et al., 2006; Warwick et al., 2005).

A small proportion of students also mentioned topics related to mental health and a quarter mentioned topics that related to both physical and mental health, demonstrating a broader understanding, as shown by a small number of previous studies (Ott et al., 2011; Wang, 2004; Ward and Wang, 1997; Natapoff, 1978). In these studies the broader views became more apparent following further discussion in focus groups (Ott et al., 2011) and interviews (Natapoff, 1978) or through the use of an open question survey, similar to the one used in this study (Ward and Wang, 1997; Wang, 2004). Notably, common broader views across the studies, including this study, included stress and fatigue (Ott et al., 2011; Ward and Wang, 1997), dealing with problems/emotions (Ward and Wang, 1997; Adler and Wahl, 1998) and non-specific mental health (Wang, 2004) (getting enough sleep/rest was classed as related to both physical and mental health in this study).

To find out more about how young people view mental health we asked them to describe what makes them mentally healthy. Despite the question being about mental 
health the most commonly mentioned topics focused on physical health, such as exercise, rest, and eating a balanced diet (which remained popular after the event, see below). This is positive as physical health is an important part of good mental health, something we highlighted in the workshop. An alternative view is that the students had trouble understanding the term 'mental health', as seen in focus groups and interviews with students in Scotland (Armstrong et al., 2000) where some responses related to the word 'health' or the word 'mental' and students had trouble relating the two. 'Health'-related responses were mostly related to eating and exercise whereas 'mental'-related responses were usually associated with mental illness despite the question being about mental health. Such a focus on illness rather than the absence of illness could be a reflection of the stigma often associated with mental illness ((Naylor et al., 2009; Corrigan and Watson, 2007) and others) or poor mental health literacy ((Friedli, 1999; Watson et al., 2004; Armstrong et al., 2000 ) and others). The positive versus negative views of mental and physical health in this study are explored in more depth in Svirydzenka et al. (2014). The majority of descriptions of mental health were positive in nature and misunderstanding of the term 'mental health' was not evident.

After physical health-related responses, 'mentally-stimulating activities' was the next most common category followed by 'positive and rational thinking', and 'social relationships'. The variety of aspects considered in response to the question 'what makes you healthy' demonstrates a fairly broad and positive view of mental health in this population, comparable with other studies (Roose and John, 2003; Natapoff, 1978; Armstrong et al., 2000).

\section{Changes in views post-intervention}

After students had completed both workshops they were surveyed again. There was a moderate change in views. When thinking about health in general students demonstrated a slight broadening of understanding with more responses related to mental health and topics related to both physical and mental health rather than just physical health.

There was more variation in views about mental health after the event suggesting that the workshop at least provoked thought about the subject. The workshop was just 45 minutes long and aimed only to raise the topic rather than address issues in detail. While the number of students mentioning physical health-related topics remained constant there was a decrease in mentions of mentally stimulating activities corresponding with an increase in mentions of positive and rational thinking, social relationships, and more than one topic. The changes seen here demonstrates a broadening of students' views to include the topics taught in the mental health workshop and suggest that short workshops such as this can improve the short-term understanding of mental health in this age group, consistent with the findings of others (Naylor et al., 2009; Pinfold et al., 2003; Pinfold et al., 2005; Watson et al., 2004; Economou et al., 2012). The intervention reported here differs from others in that it aimed to increase young people's inclusion of mental health as an important component of general health rather than to just improve knowledge and attitudes. Improvements in factual knowledge and attitudes have previously been shown following similarly short programmes (Pinfold et al., 2003; Pinfold et al., 2005) so it does seem that short educational workshops can have a positive effect, at least in the short term.

Other school-based interventions taking place over a slightly longer period (5-6 lessons) have also led to improvements in knowledge and attitudes (Naylor et al., 2009; 
Watson et al., 2004) and it is possible that a more sustained intervention over a longer period would have shown a greater broadening of attitudes here. Whether the changes in knowledge and attitude are sustained over time is largely unknown and underexplored (Wyn et al., 2000). The few studies that have conducted mid-term follow-up (3-12 months after the intervention) report conflicting results. Naylor et al., (2009) (Naylor et al., 2009) reported an improvement in factual knowledge (the ability to name five mental health difficulties) measured six months after their intervention whereas Pinfold et al. (2003) (Pinfold et al., 2003) found that factual recall of information about mental health problems was lost after six months. Both studies did find that attitude improvements were maintained. Other studies focusing more on stigma report some maintenance of attitude change but not of social distance, i.e. the willingness of students to associate with people with mental health problems (Conrad et al., 2009; Economou et al., 2012). Sustained improvements in beliefs and attitudes are most desired from a health promotion point of view as they contribute to self-efficacy in healthy living. Overall, the longer-term impact of school-based interventions seems to vary depending on the intervention content and evaluation methods. It is likely that the attitudes and beliefs of young people would also be influenced by external factors over time, as well as being influences by the effects of age (Wang, 2004; Natapoff, 1978), therefore longer-term follow-up is not without difficulties. None-the-less, follow-up at a later date would be useful in studies with an appropriate control group.

\section{Gender differences in views about health and response to educational interventions}

Studies have suggested that girls have a broader view of health than boys (Wang, 2004; Blaxter, 2004), particularly regarding the inclusion of mental health (Cotton et al., 2006). This was also seen prior to our intervention, where significantly more girls mentioned topics related to both physical and mental health when asked to describe general health. However, the proportion mentioning mental health was the same for both genders, in contrast to the finding that more boys considered mental health to be part of general health by Wang (2004) [18].

When looking at specifically at mental health descriptions there was little difference between the two genders, as seen previously (Villeneuve et al., 1996). The only difference observed here was the higher proportion of girls mentioning topics related to social relationships both before and after the event. This is an interesting finding as there has been little research into gender differences in knowledge and attitudes to mental health in young people, except for the finding that feeling fit is more important for girls and personal achievement is more important for boys when thinking about what makes them healthy (Armstrong et al., 2000).

\section{Limitations}

Assessment of the impact of this study and the ability to make general recommendations from the results is limited by several factors, specifically the lack of longer-term follow up, the small sample size with limited demographic diversity, the absence of a control group, and the lack of in-depth questioning of students. Such factors were beyond the scope of this study as we sought to deliver a novel and pragmatic pilot intervention to enable us to explore young people's views. While we show some positive changes, we do not know if 
these were sustained. Any future work should try and have longer term follow up. Individual demographics of the participants in this study are not known and pre- and postevent questionnaires were anonymous and not linked. It would be interesting to repeat the survey with more ethnicity information and a larger and more diverse group of students, as a study with Scottish high school students suggests a more positive and clearer perception of mental health from students in suburban schools and minority ethnic groups compared to the most deprived and rural areas, although the sample size was too small for conclusions to be drawn (Armstrong et al., 2000). Further qualitative study would also be beneficial to allow a more in-depth analysis of what young people think and to see if there is any difference in the opinions expressed through an interview or focus group versus an anonymous written questionnaire. Our study shows that short interactive workshops have the potential to increase knowledge about health and also provoke thought about the issues but the fact that they were delivered as part of an outreach project has some limitations in that it may be difficult to apply them in other contexts.

\section{Conclusion}

Despite these limitations, the present study contributes to our understanding of adolescents' views about health and wellbeing. Such understanding will enable better tailoring of health education and promotion interventions, which has not often been considered in the planning of mental health promotion interventions to date (Oliver et al., 2008). Reports in England and from the World Health Organization suggest the need to educate adolescents and facilitate their ability to make responsible choices for themselves and their health (DCSF, 2009; DES, 2005; WHO, 1997). The short and contained school time intervention evaluated here was moderately successful in improving the perception of mental health as a key aspect of wellbeing. This demonstrates that such a one-off approach could be beneficial in broadening the perspectives of adolescents. Further developments of this approach, such as lengthening the 'Healthy Mind' workshop, could help to deepen understanding. The implications for policy are limited but may indicate that polices relating to health education may usefully consider the approach described here in engaging schools and students to begin to discuss health and especially mental health.

\section{Acknowledgments}

The authors would like to thank Sophia Kosmider for help with the literature review and Julian Barwell for contribution to the intervention design.

\section{Funding}

This work was supported by a grant from The National Institute for Health Research Collaboration for Leadership in Applied Health Research and Care - Leicestershire, Northamptonshire and Rutland (NIHR CLAHRC for LNR), UK. 


\section{References}

Adler AK and Wahl OF. (1998) Children's Beliefs About People Labeled Mentally III. American Journal of Orthopsychiatry 68: 321-326.

Armstrong C, Hill M and Secker J. (2000) Young people's perceptions of mental health. Children \& Society 14: 60-72.

Blaxter M. (2004) Health and lifestyles: London: Routledge.

Bremner P, Dalziel D and Evans L. (2006) Evaluation of the 5 A Day Programme - Final Report.

Brown T and Summerbell C. (2009) Systematic review of school-based interventions that focus on changing dietary intake and physical activity levels to prevent childhood obesity: an update to the obesity guidance produced by the National Institute for Health and Clinical Excellence. Obesity Reviews 10: 110-141.

Conrad I, Dietrich S, Heider D, et al. (2009) "Crazy? So what!":: A school programme to promote mental health and reduce stigma - results of a pilot study. Health Education 109: 314-328.

Corrigan PW and Watson AC. (2007) How Children Stigmatize People With Mental Illness. International Journal of Social Psychiatry 53: 526-546.

Cotton SM, Wright A, Harris MG, et al. (2006) Influence of Gender on Mental Health Literacy in Young Australians. Australian and New Zealand Journal of Psychiatry 40: 790-796.

DCSF (2009) Your Child, Your Schools, Our Future: Building a 21st Century Schools System. Department for Children, Schools and Families (DCSF), UK.

DES (2005) Every Child Matters: An Education Strategy. Department of Education and Skills (DES), UK.

Dogra, N., Svirydzenka, N., Dugard, P., Singh, S., \& Vostanis, P. (2013). Characteristics and rates of mental health problems among Indian and White adolescents in two English cities. British Journal of Psychiatry, 202, 1-7.

DoH (2010) Change4Life One Year On. HMG Department of Health, UK, 104.

DoH (2011) No Health Without Mental Health: A Cross-Government Mental Health Outcomes Strategy for People of All Ages. HMG Department of Health, UK.

Economou M, Louki E, Peppou LE, et al. (2012) Fighting psychiatric stigma in the classroom: The impact of an educational intervention on secondary school students' attitudes to schizophrenia. International Journal of Social Psychiatry 58: 544-551.

Friedli L. (1999) From the Margins to the Mainstream: The Public Health Potential of Mental Health Promotion. Journal of Public Mental Health 1: 30-36.

Grace M, Woods-Townsend K, Griffiths J, et al. (2012) Developing teenagers' views on their health and the health of their future children. Health Education 112: 543-559.

Green H, McGinnity A, Meltzer H, Ford T, Goodman R. Mental Health of Children and Young People in Great Britain, 2004. Office for National Statistics, 2005.

Jané-Llopis E, Barry M, Hosman C, et al. (2005) Mental health promotion works: a review. Promotion \& Education 12: 9-25.

Jokela M, Ferrie J, Kivimäki M (2009) Childhood problem behaviors and death by midlife: The British National Child Development Study. Journal of the American Academy of Child and Adolescent Psychiatry 48: 19-24.

Lopez AD, Mathers CD, Ezzati M, et al. (2006) Global and regional burden of disease and risk factors, 2001: systematic analysis of population health data. The Lancet 367: 17471757. 
Natapoff JN. (1978) Children's views of health: a developmental study. American Journal of Public Health 68: 995-1000.

Naylor PB, Cowie HA, Walters SJ, et al. (2009) Impact of a mental health teaching programme on adolescents. British Journal of Psychiatry 194: 365-370.

Oliver S, Harden A, Rees R, et al. (2008) Young people and mental health: novel methods for systematic review of research on barriers and facilitators. Health Education Research 23: 770-790.

Ott MA, Rosenberger JG, McBride KR, et al. (2011) How Do Adolescents View Health? Implications for State Health Policy. Journal of Adolescent Health 48: 398-403.

Pinfold V, Stuart H, Thornicroft G, et al. (2005) Working with young people: the impact of mental health awareness programs in schools in the UK and Canada. World Psychiatry 4: 48-52.

Pinfold V, Toulmin H, Thornicroft G, et al. (2003) Reducing psychiatric stigma and discrimination: evaluation of educational interventions in UK secondary schools. The British Journal of Psychiatry 182: 342-346.

Roose GA and John AM. (2003) A focus group investigation into young children's understanding of mental health and their views on appropriate services for their age group. Child: Care, Health and Development 29: 545-550.

Svirydzenka, N., Bone, C., Dogra, N. (2014). Schoolchildren's perspectives on the meaning of mental health. Journal of Public Mental Health, 13: 4 - 12

Villeneuve C, Berube $H$, Ouellet R, et al. (1996) Prevention concerning mental health: the adolescent's perspective. Canadian Journal of Psychiatry 41: 392-399.

von Stumm S, Deary IJ, Kivimäki, M, et al. (2011) Childhood behavior problems and health at midlife: 35-year follow-up of a Scottish birth cohort. Journal of Child Psychology and Psychiatry 52: 992-1001.

Wang W. (2004) Mainland Chinese Students' Concept of Health. Asia-Pacific Journal of Public Health 16: 89-94.

Ward L and Wang W. (1997) Adolescents' concept of health: A phenomenological study of adolescents in Rockhampton, Queensland, in The Abstracts of the 10th Australasian Human Development Conference 10-12 July 1997 Adelaide, South Australia. Australian Journal of Psychology 49: 76.

Warwick I, Aggleton P, Chase E, et al. (2005) Evaluating healthy schools: perceptions of impact among school-based respondents. Health Education Research 20: 697-708.

Watson AC, Otey E, Westbrook AL, et al. (2004) Changing Middle Schoolers' Attitudes About Mental Illness Through Education. Schizophrenia Bulletin 30: 563-572.

White B, Nicholls D, Christie D, et al. (2012) Childhood psychological function and obesity risk across the lifecourse: findings from the 1970 British Cohort Study. International Journal of Obesity 36: 511-516.

WHO (1997) The World Health Report: Conquering Suffering Enriching Humanity. World Health Organisation, Geneva.

WHO (2003) Caring for Children and Adolescents with Mental Disorders: Setting WHO Directions. World Health Organization, Geneva.

WHO (2005) Atlas on Child and Adolescent Mental Health Resources - Global Concerns: Implications for the Future 2005. World Health Organization.

Wyn J, Cahill H, Holdsworth R, et al. (2000) MindMatters, a Whole-School Approach Promoting Mental Health and Wellbeing. Australian and New Zealand Journal of Psychiatry 34: 594-601. 
Table 1. Students' descriptions of what makes them generally healthy and mentally healthy before and after the event - percentage of students mentioning each topic.

\begin{tabular}{|c|c|c|c|}
\hline & & $\begin{array}{l}\text { Before }(\% \\
\text { students) }\end{array}$ & $\begin{array}{l}\text { After }(\% \\
\text { students) }\end{array}$ \\
\hline \multicolumn{4}{|l|}{ What makes someone healthy? } \\
\hline Mentioned physical health-related topics & Total $^{\mathrm{a}}$ & 100 & $97^{\mathrm{b}}$ \\
\hline Mentioned mental health-related topics & Total & 8 & 12 \\
\hline $\begin{array}{l}\text { Mentioned topics relating to both physical and } \\
\text { mental health }\end{array}$ & Total & 25 & 19 \\
\hline \multicolumn{4}{|l|}{ What makes you mentally healthy? } \\
\hline Mentioned physical health-related topics & Total & 43 & 37 \\
\hline Mentioned topics related to social relationships & Total & 20 & $37^{c}$ \\
\hline $\begin{array}{l}\text { Mentioned topics related to mentally stimulating } \\
\text { activities }\end{array}$ & Total & 35 & $23^{b}$ \\
\hline $\begin{array}{l}\text { Mentioned topics related to positive and rational } \\
\text { thinking }\end{array}$ & Total & 32 & $57^{c}$ \\
\hline $\begin{array}{l}\text { Mentioned topics related to avoiding stressful } \\
\text { situations }\end{array}$ & Total & 17 & 24 \\
\hline Mentioned other topics & Total & 3 & 7 \\
\hline Mentioned more than one topic & Total & 44 & $62^{\mathrm{c}}$ \\
\hline
\end{tabular}

a 'Total' includes those students who did not state their gender.

${ }^{b}$ Significant differences from before value, $\mathrm{P}<0.05$ Pearson Chi Square Test for Independence with Yates' Continuity Correction.

' Significant differences from before value, $\mathrm{P} \leq 0.001$ Pearson Chi Square Test for Independence with Yates' Continuity Correction. 
Table 2. Gender differences in students' descriptions of what makes them generally healthy and mentally healthy before and after the event - percentage of female and male students mentioning each topic.

\begin{tabular}{llll}
\hline & & $\begin{array}{l}\text { Before (\% } \\
\text { students) }\end{array}$ & $\begin{array}{l}\text { After (\% } \\
\text { students) }\end{array}$ \\
\hline What makes someone healthy? & & & \\
Mentioned physical health-related topics & Female & 100 & 98 \\
& Male & 100 & $93^{\text {a,d }}$ \\
Mentioned mental health-related topics & Female & 9 & 15 \\
& Male & 8 & 12 \\
Mentioned topics relating to both physical and & Female & 35 & 27 \\
mental health & Male & $14^{\mathrm{c}}$ & 15 \\
What makes you mentally healthy? & & & \\
Mentioned physical health-related topics & Female & 41 & 32 \\
& Male & 46 & 48 \\
Mentioned topics related to social relationships & Female & 27 & $48^{\mathrm{b}}$ \\
& Male & $9^{\mathrm{c}}$ & $22^{\mathrm{a}, \mathrm{c}}$ \\
Mentioned topics related to mentally stimulating & Female & 30 & 21 \\
activities & Male & 39 & 22 \\
Mentioned topics related to positive and rational & Female & 37 & $66^{\mathrm{b}}$ \\
thinking & Male & 33 & $52^{\mathrm{a}}$ \\
Mentioned topics related to avoiding stressful & Female & 19 & 28 \\
situations & Male & 15 & 19 \\
Mentioned other topics & Female & 4 & 7 \\
& Male & 3 & 7 \\
Mentioned more than one topic & Female & 49 & $73^{\mathrm{b}}$ \\
& Male & 40 & 57 \\
\hline Significant differences from before value, P<0.05 & Pearson Chisquare Test & \\
& & &
\end{tabular}

${ }^{\mathrm{a}}$ Significant differences from before value, $\mathrm{P}<0.05$ Pearson Chi Square Test for Independence with Yates' Continuity Correction.

${ }^{b}$ Significant differences from before value, $\mathrm{P}<0.01$ Pearson Chi Square Test for Independence with Yates' Continuity Correction.

' Significant differences from female value, $\mathrm{P}<0.01$ Pearson Chi Square Test for Independence with Yates' Continuity Correction.

${ }^{d}$ Fisher's Exact Probability Test was used were there were more than $20 \%$ of cells with expected counts less than 5 .

NA - Value not computed due to there being no difference. 
Table 3. Examples of students' mental health-related descriptions of what makes them generally healthy after the event.

What makes someone healthy?

"Good attitude and thinking" (boy)

"Avoiding stress" (girl)

"Being happy, feeling good about yourself and not being stressed" (girl)

"Being around friends and family" (girl) 
Table 4. Examples of students' descriptions of what makes them mentally healthy.

\section{Before}

\section{Positive and} rational

thinking

Social

relationships (girl)

\section{After}

"Having a positive attitude towards life" (boy)

"Being happy and having positive thoughts" (girl) attitude and think clearly" (boy) "Staying positive inside yourself and keeping your mind clear"

"Having friends you can trust" (girl)
"Being able to tell someone how you
feel" (girl)
"Not bottling up your emotions" (girl)
"Being able to make friends easily" (girl)
"Having a good social life" (girl)
"Having a good home environment" (girl)
"Being around family and friends" (girl)


Box 1. Content of the workshops.

'Health Body' - 85 minutes interactive activities about healthy eating and physical health.

Activities:

Short introductory presentation

Breakfast quiz using voting keypads

Breakfast cereal nutritional label activity - ordering cereals by sugar, protein and fibre content Breakfast facts and consequences card matching game

Experiment to see how much fat is in crisps

Calculating the amount of sugar in some junk food snacks

Computer activity looking at energy levels

Calculating the exercise equivalent of calories in food

Lung function testing

Body fat testing

'Healthy Mind' - 45 minute session exploring the meaning of the term 'Mental Health' and what helps young people to stay mentally healthy.

Each workshop consisted of 6-8 small groups

Half of the groups were given the question "What does the term 'Mental Health' mean?" and the other half "What do you think helps young people to stay mentally healthy?"

To encourage independent debate, groups with the same questions sat separately.

Students received 10-15 minutes of discussion time to write their answers on the blank poster sheets provided.

Concluded the workshop by encouraging the students to compare their thoughts with the Bright Futures definition of mental health

'Healthy Mind' section was developed from Svirydzenka et al. (2014) 